\title{
A 21-year-old male with throat swelling and difficulty swallowing
}

\author{
Patrick K. Gleeson ${ }^{1}$, Theodore E. Kelbel ${ }^{2}$, Timothy J. Craig*2 \\ ${ }^{1}$ Internal Medicine Residency Program, Temple University, Philedelphia, USA \\ ${ }^{2}$ Allergy \& Immunology Fellowship Program, Penn State Hershey Medical Center, Hershey, USA
}

Received: November 18, 2015

Accepted: December 22, 2015 Online Published: December 29, 2015

DOI: $10.5430 /$ crim.v3n1p47

URL: http://dx.doi.org/10.5430/crim.v3n1p47

\begin{abstract}
Background: Angioedema is an extravasation of fluid into cutaneous, subcutaneous, or mucosal tissues. The condition has a wide range of clinical presentations and possible etiologies. Isolated uvular angioedema, also termed Quincke's disease, may be idiopathic or a manifestation of hereditary angioedema, a medication reaction, food allergy, infection, trauma, inhalation, or general anesthesia.

Case description: We report on a patient who presented to the emergency department with throat swelling and difficulty swallowing. The patient was admitted due to concern for epiglottitis and airway compromise. The patient quickly improved after receiving doses of an oral antihistamine and an inhaled corticosteroid and was discharged the next day. On assessment for etiology he had a very unlikely cause for his swelling.

Clinical implications: Angioedema of the oropharyngeal area is concerning for the development of airway compromise and may require laryngoscopy. A thorough history, physical exam, and laboratory investigation differentiate angioedema from other causes of airway edema.
\end{abstract}

Key Words: Quincke's angioedema, Corn allergy, Uvular angioedema

\section{INTRODUCTION}

Quincke's disease refers to isolated uvular angioedema due to any cause. Angioedema is localized swelling of dermal, mucosal, and submucosal tissues due increased vascular permeability and movement of fluid into the interstitial space. This contrasts with urticaria, a swelling of the dermis and epidermis only. These two conditions have frequently overlapping causes and clinical findings. The astute clinician should be able to recognize the differences, however, particularly in the setting of acute airway edema or other emergent presentations. This is because urticaria, or urticaria and angioedema together, are frequently histamine-mediated, whereas isolated angioedema is in many cases caused by increased bradykinin and may have a different approach to treatment. When angioedema appears to be isolated to the uvula, the clinician must also consider local insults to the uvula in the form of thermal injury, inhalation of an irritant or physical trauma, especially from snoring.

Regardless, the first step in evaluation of swelling in the vicinity of the upper respiratory tract is to monitor breathing and secure the airway if necessary. Empiric medical treatment may be warranted until a detailed history, physical examination, and laboratory investigation reveals the diagnosis and tailors acute and long-term medical treatment. In our case

\footnotetext{
*Correspondence: Timothy J. Craig, DO; Email: tcraig@hmc.psu.edu; Address: Penn State Hershey Medical Center, 500 University Drive, Mail Code h041, Hershey, PA 17033, USA.
} 
report, we describe a patient with idiopathic angioedema of the uvula who successfully responded to antihistamine and corticosteroid therapy, but was later found to have a unique etiology for his angioedema.

\section{Case presentation}

A 21-year-old male with a history of a shellfish allergy presented to the emergency department with a sensation of throat swelling and difficulty swallowing. He awoke with the symptoms that morning and saw significant uvular swelling in the mirror, prompting a visit to the emergency department. He did have a choking sensation when he woke up, but thereafter denied any difficulty breathing. He also denied hives, itching, wheezing, fevers, throat pain, voice changes, or vomiting. He reported ingesting $200 \mathrm{mg}$ ibuprofen approximately 9 hours before symptom onset. He also ate a chicken quesadilla made with corn tortillas 4-5 hours before waking up. He was not on any regular medications and has never been on an ACE inhibitor. He did have one previous episode of throat swelling after eating crab legs 2 years prior, but never had allergy testing prior to our evaluation. He had no known medication allergies. He denied any other past history of throat swelling, but said that his uvula was noted to be large on previous physical exams. He denied any history of tobacco, illicit drug use, heartburn or reflux symptoms. His past medical history was otherwise notable only for sickle cell trait. There was no family history of angioedema. His vital signs were within normal limits. Physical exam showed swelling of the uvula without erythema. The skin exam showed no rashes or hives and the rest of the physical exam was normal.

A soft tissue X-ray of the neck showed no frank "thumb print" sign, and the nasopharynx, oropharynx, hypopharynx, and prevertebral soft tissues were normal. A flexible fiberoptic exam revealed a normal oropharynx except for uvular edema and base of the tongue tonsillar hypertrophy. There was no subglottic edema. His CBC and BMP were unremarkable. IgE was high at 458 and tryptase was normal. $\mathrm{C} 4$ was normal. He was admitted to the internal medicine service over concern for the development of airway compromise. He was started on IV dexamethasone and diphenhydramine and experienced complete clinical recovery overnight. He was discharged on a short course of oral prednisone, cetirizine, and an injectable epinephrine.

In outpatient allergy follow up, corn allergy was suspected with positive skin ( $5 \mathrm{~mm}$ wheal by $8 \mathrm{~mm}$ flare) and specific $\operatorname{IgE}(0.74 \mathrm{kU} / \mathrm{L})$ testing. Additionally, his crab allergy was confirmed with specific IgE, $1.26 \mathrm{kU} / \mathrm{L}$ as well as showing the presence of IgE to similar crustaceans shrimp, $1.88 \mathrm{kU} / \mathrm{L}$, and lobster, $1.16 \mathrm{kU} / \mathrm{L}$. The time sequence from consumption of the corn to swelling was longer than usual, but he did fall asleep shortly after eating the quesadilla, so the onset of his symptoms may have been sooner (within the 30 minute- 2 hour range classic for IgE mediated food reactions). It is possible the uvular swelling needed to reach a critical size to cause arousal from the sleeping state. He has avoided corn since the testing without a return of his symptoms. Prior to the incident, it was not clear how much corn he had been eating in his diet but he reported not regularly eating corn products. Although corn allergy would be odd to develop later in life, the positive testing, reaction and lack of other offending triggers makes it the most likely etiology for his symptoms. Other possible etiologies could be an irritant or allergic reaction to the spices in the food, or possible thermal injury induced edema. Both seem less likely given the positive corn allergy evaluation, lack of repeat symptoms despite not avoiding spices and no patient report of the sensation of thermal injury while eating. Definitive testing of corn oral challenge was offered to the patient but declined given fear of repeat throat swelling symptoms. Injectable epinephrine 0.3 $\mathrm{mg}$ for intramuscular use during anaphylaxis was prescribed for accidental ingestion.

\section{DisCUSSION}

After securing and monitoring the airway, possibly performing a laryngoscopy, and administering empiric medical treatment to decrease swelling, a thorough history and physical exam and targeted laboratory testing may help identify a cause. Edema of the uvula is angioedema and not urticaria given the mucosal involvement, but the question remains whether urticaria is present elsewhere, which would strongly suggest a type I hypersensitivity reaction. Angioedema is best categorized as either histamine or bradykinin induced. Histamine induced may be caused by mast cell and basophil release of histamine and may be localized or systemic when part of an anaphylactic reaction. In contrast, bradykinin induced angioedema is not allergic, but secondary to over stimulation of the contact system and either over production or decreased catabolism of bradykinin. Angioedema is typically described as nonerythematous, nonpitting, nonpruritic swelling in the mucosal areas, throat, face, hands, genitals, and gastrointestinal tract. An angioedematous uvula is pale and is similar in appearance to a white grape. ${ }^{[1]} \mathrm{Ur}-$ ticaria, meanwhile, presents as erythematous, pruritic wheals that can manifest anywhere on the body but spares mucosal surfaces. ${ }^{[2]}$

A clinical presentation of isolated angioedema may still be allergic but also suggests other possibilities. Nonallergic angioedema may also involve mast cells, as in the case of some drugs and radiocontrast agents, but as noted above may also be due to increased production or decreased catabolism 
of bradykinin. This latter mechanism is seen in hereditary-, acquired-, and ACE inhibitor induced angioedema. A third category of angioedema is idiopathic and is a diagnosis of exclusion. Although the pathophysiology is unknown, idiopathic angioedema has been observed to be associated with various stressors such as heat, cold, vibration, pressure, or exercise. ${ }^{[3]}$ Passive heating or active heating may cause an acute cholinergic hypersensitivity reaction. ${ }^{[4]}$ Quincke's disease may be due to any of these causes or as a result of a direct insult to the uvula, which can include trauma, infection, general anesthesia, snoring induced vibration or inhalation exposure. ${ }^{[5]}$

\subsection{Clinical manifestations}

Quincke's angioedema is by definition isolated to the uvula, sparing other common areas for angioedema. The patient's history will provide clues to the etiology, although frequently the cause is idiopathic and no trigger can be identified. The onset of the swelling occurs over minutes to hours and lasts for hours to several days. Patients may complain of fullness in the throat and dysphagia. In severe cases, signs of airway obstruction may be present. ${ }^{[4]}$ Patients must be closely monitored early on in the presentation, as the rapid development of airway compromise may ensue.

\subsection{Diagnosis}

Angioedema is a clinical diagnosis, and idiopathic angioedema is a diagnosis of exclusion. Clinicians should inquire about precipitating events prior to the onset of angioedema, including foods consumed, inhalation of any irritants, medications, and snoring. Particular focus should be paid to the onset of the event following ingestion of food, as IgE-mediated food allergies typically present within minutes up to 2 hours after ingestion. The clinician should undergo a thorough drug history to rule out a possible offending medication. The time course for medications is similarly important. For example, NSAID-induced angioedema (IgE-mediated or nonallergic) and ACE inhibitor-induced angioedema begin minutes to several hours after ingestion. ${ }^{[6]}$ The vital signs and physical examination are important in pinpointing the cause. Fever, erythema, or pain will suggest uvulitis over angioedema, and pruritis, urticaria, flushing, or bronchospasm are more indicative of mast cell activation present in an allergic reaction.

After an initial evaluation, laboratory testing may yield a diagnosis, but frequently no etiology can be determined. A CBC with differential, CRP, and ESR should be normal in the absence of infection. The complement protein $\mathrm{C} 4$ will screen for $\mathrm{C} 1$ inhibitor deficiency, and will be within the normal range in idiopathic angioedema. A low $\mathrm{C} 4$ is sen- sitive for hereditary angioedema with low of dysfunctional $\mathrm{C} 1$-inhibitor and acquired angioedema, and if $\mathrm{C} 4$ is normal during an acute episode of angioedema these diagnoses are virtually excluded. ${ }^{[7]}$ Tryptase is not particularly helpful unless anaphylaxis is suspected. If a food allergen is suspected, allergen-specific skin testing and/or specific IgE immunoassays may be performed.

\subsection{Differential diagnosis}

The differential diagnosis for angioedema of the uvula includes hereditary and acquired angioedema, food allergy, irritation due to chemical inhalation or snoring, drug reaction, infection, and neoplasm. The clinician must be careful to distinguish angioedema from another acute inflammatory process of the uvula. Uvulitis must be considered in a young febrile patient presenting with a swollen uvula, most commonly due to group A streptococcal pharyngitis. Many drugs, acting via a variety of different mechanisms, can cause acute uvular swelling. Common triggers include aspirin, NSAIDs, opioids, and ACE inhibitors. While many responsible drugs act via mast cell activation and would not be expected to cause a first-time drug reaction in a chronic user, it is important to know that ACE inhibitors can cause angioedema spontaneously even after years of use. ${ }^{[8]}$

\subsection{Management and prognosis}

The first priority in a patient presenting with uvular edema is immediate assessment and management of the airway. Nearby structures, including the epiglottis and larynx, may become involved and cause progressive obstruction of the airway necessitating intubation. A laryngoscopy or soft tissue lateral radiograph of the neck is warranted to rule out hypopharyngeal swelling not evident on the physical exam, but intubation when needed should not be delayed for this procedure. ${ }^{[1]}$ Empiric medical treatment may consist of antihistamines, epinephrine, and corticosteroids. Patients should be advised to avoid known triggers if possible. In cases, where hereditary angioedema is confirmed, appropriate bradykinin cascade therapy is indicated: replacement of $\mathrm{C} 1$ esterase inhibitor protein, bradykinin receptor antagonist (icatibant), or kallikrein inhibitor (ecallantide). ${ }^{[9]}$ A recent publication has also shown some benefit of icatibant in airway threatening ACE inhibitor induced angioedema. ${ }^{[10]}$

\section{Conclusion}

Quincke's disease, also known as isolated uvular angioedema is an interesting clinical presentation of a potentially life threatening condition with a broad differential diagnosis. Astute recognition of the problem and stabilization of the airway are the universal first steps in the care of this condition, however, attention to detail in the history and other parts of 
the physical exam will allow for the correct diagnosis and allergy described in the literature. appropriate treatment. We believe our patient is the only identified case of Quincke's angioedema secondary to corn

\section{REFERENCES}

[1] Goldberg R, Lawton R, Newton E, et al. Evaluation and management of acute uvular edema. Annals of Emergency Medicine. 1993; 22(2): 251-255. http://dx.doi.org/10.1016/S0196-0 644 (05) 80215-X

[2] Muller BA. Urticaria and angioedema: a practical approach. American Family Physician. 2004; 69(5): 1123-1128. PMid:15023012

[3] Lewis LM. Angioedema: etiology, pathophysiology, current and emerging therapies. The Journal of Emergency Medicine. 2013; 45(5): 789-796. PMid:23992848 http://dx.doi.org/10.1016 /j.jemermed.2013.03.045

[4] Mattingly G, Rodu B, Alling R. Quincke's disease: nonhereditary angioneurotic edema of the uvula. Oral Surgery, Oral Medicine, Oral Pathology. 1993; 75(3): 292-295. http://dx.doi.org/10.1016 /0030-4220 (93) 90139-U

[5] Johnson W, Shastri N, Fowler M. Quincke's disease. Western Journal of Emergency Medicine: Integrating Emergency Care with Population Health. 2011; 12(4): 370. PMid:22224120 http://dx.doi .o rg/10.5811/west jem.2011.3.2246
[6] Banerji A, Sheffer AL. The spectrum of chronic angioedema. Allergy and Asthma Proceedings. 2009; 30(1): 11-16. PMid:19331715 http://dx.doi.org/10.2500/aap.2009.30.3188

[7] Gompels MM, Lock RJ, Morgan JE, et al. A multicenter evaluation of the diagnostic efficiency of serological investigations for $\mathrm{C} 1$ inhibitor deficiency. Journal of Clinical Pathology. 2002; 55(2): 145-147. PMid:11865013 http://dx.doi.org/10.1136/jcp.55.2.145

[8] Temiño VM, Peebles RS Jr. The spectrum and treatment of angioedema. The American Journal of Medicine. 2008; 121(4): 282286. PMid:18374684 http://dx.doi.org/10.1016/j.amjmed. 2007.09 .024

[9] Craig T, Pürsün EA, Bork K, et al. WAO guideline for the management of hereditary angioedema. WAO Journal. 2012; 5: 182-199. http://dx.doi.org/10.1097/wox.0b013e318279affa

[10] Bas M, Greve J, Stelter K, et al. A randomized trial of icatibant in ACE-inhibitor-induced angioedema. New England Journal of Medicine. 2015; 372(5): 418-425. PMid:25629740 http: //dx.doi.org/10.1056/NEJMoa1312524 